\title{
RAPIDLY PROGRESSIVE INTERSTITIAL LUNG DISEASE IN A DERMATOMYOSITIS PATIENT SUCCESSFULLY TREATED WITH TRIPLE
} \section{THERAPY}

Bruno Bordallo Corrêa ${ }^{1, \star}$, Hany Kelly Cruz ${ }^{1}$, Júlia Yoneshigue Laranja de Oliveira ${ }^{1}$, Lizeth Chaparro Del Portillo , Luiza Catharina Brussasco Grandini $^{1}$, Marcus Vinicius de Jesus da Silva ${ }^{1}$, Carla da Fontoura Dionello ${ }^{1}$, Maria Isabel Dutra Souto ${ }^{1}$, Julio Cesar Borges ${ }^{1}$, Mirhelen Mendes Abreu$^{1}$, Blanca Elena Rios Gomes Bica ${ }^{1}$

1.Universidade Federal do Rio de Janeiro, Rio de Janeiro (RJ), Brazil.

*Corresponding author: brunobaf@hotmail.com

\section{BACKGROUND}

Interstitial lung disease is a serious manifestation of inflammatory myopathies, especially dermatomyositis. Pulmonary disease associated with myopathies has a wide spectrum of severity. Rapidly progressive pulmonary interstitial disease (RP-ILD) is closely associated with the anti-MDA5 antibody and hyperferritinemia. The clinical phenotype associated with this antibody is very characteristic and involves non-healing alopecia, ulcerated palmar papules and RP-ILD. Prompt recognition and diagnosis of RP-ILD associated with dermatomyositis is essential for initiating aggressive treatment.

\section{CASE REPORT}

Here we report a 44-year-old woman who developed progressive dyspnea 8 months before hospital admission. She also developed 5 months before admission, the appearance of skin lesions such heliotrope, shawl sign, diffuse rash, palmar papules, noncicatricial alopecia and Gottron's sign (Fig. 1). She mentioned occasional fever, polyarthralgia and weight loss of about $20 \mathrm{~kg}$ in the period. She denied dysphagia, productive cough, chills or other symptoms. In admission, she was vigilant, conscious, had the

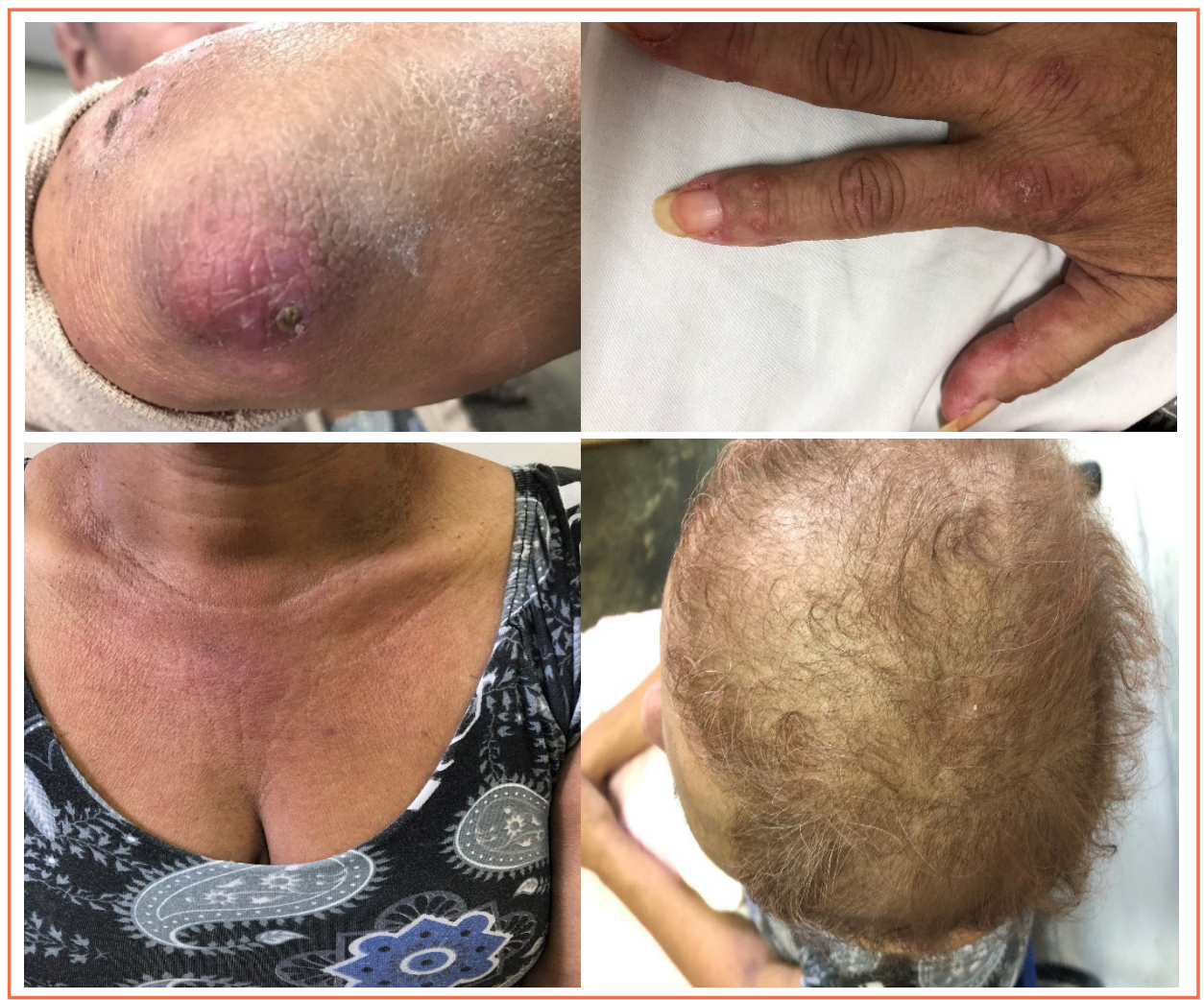

Figure 1. Skin manifestations. 
skin lesions described above and severe dyspnea. No muscle weakness was found on examination. She was hypoxemic, saturating $84 \%$ at room air. In laboratorial exams, she had mild anemia, normal muscle enzymes and negative FAN, Jo-1 and Mi-2 antibodies and a very high ferritin ( $2281 \mathrm{ng} / \mathrm{dL}$ ). High resolution chest tomography showed diffuse ground glass opacities, predominantly in peripheral distribution and perilobular opacities (Fig. 2). Due to her skin manifestations, amyopathic presentation, hyperferritinemia and lung disease, we assume that it could be an anti-MDA5 RP-ILD dermatomyositis. Unfortunately, it was not possible to dose the antibody because the institutional laboratory did not perform the exam. Due to severity of the anti-MDA5 dermatomyositis RP-ILD, we started triple-therapy with pulse therapy with methylprednisolone for 3 days associated with cyclophosphamide $500 \mathrm{mg}$ every two weeks and intravenous cyclosporine. Her treatment was stopped for 2 weeks due to staphylococcal pyomyositis. The patient evolved with significant clinical and radiological improvement and was discharged in 60 days.

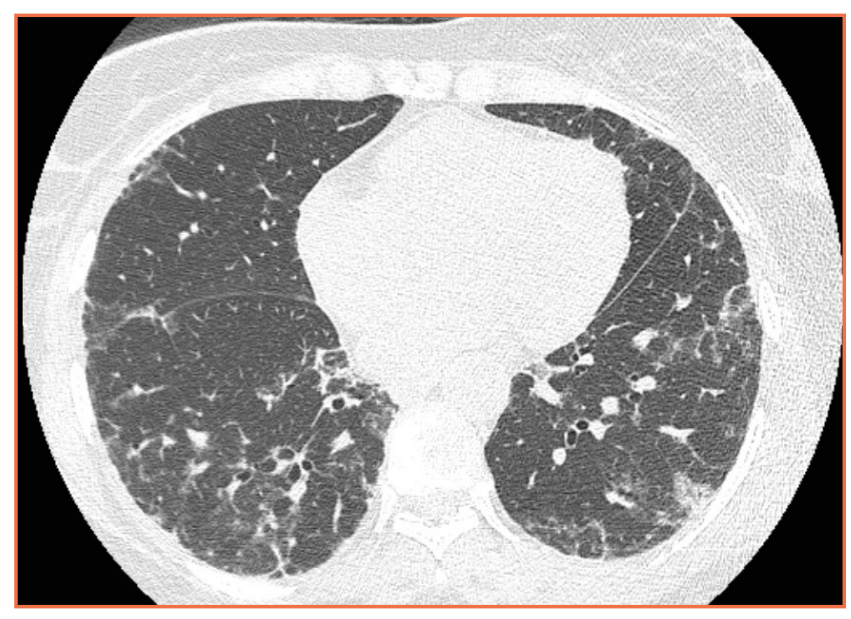

Figure 2. Chest tomography.

\section{CONCLUSION}

Rapidly progressive pulmonary interstitial disease associated with dermatomyositis, particularly those associated with antiMDA5 antibody, is a serious and life-threatening manifestation. The knowledge of this form of pulmonary involvement as well as the clinical characteristics associated with the anti-MDA5 antibody helps in the early diagnosis. Prompt treatment of RP-ILD is critical to improving mortality. 